\section{Acquisition of}

DuPont Performance

\section{Coatings by}

The Carlyle Group

\section{Completed}

The Carlyle Group, a globally active asset management company, has completed the acquisition of DuPont Performance Coatings for $\$ 4.9$ billion and has announced that the company is being renamed Axalta Coating Systems. Axalta Coating Systems is a global supplier of coatings to the transportation and industrial sectors. "We are excited to invest in Axalta Coating Systems and believe its strong market position and global footprint will enable the company to capitalize on opportunities in rapidly emerging markets such as China and Brazil. As experienced investors in the industrial and transportation sectors, the One Carlyle global network can help Axalta Coating Systems grow and create value," said Martin Sumner, Principal of The Carlyle Group. As an independent company, Axalta Coating Systems will build on a foundation of more than 90 years in the coatings industry. The company serves more than 120,000 customers in 130 countries. Its market position is supported by global brands such as Standox, Spies Hecker, DuPont Refinish, Alesta Powder Coatings, Nap-Gard FBE and Abcite Powder Coatings. Axalta Coating Systems will continue to do business as DuPont Performance Coatings for the next few months until the new name is formally launched in the second quarter of 2013.

\title{
Heraeus Noblelight Acquires Fusion UV
}

Leraeus Noblelight, a business - group of the precious metal and technology group Heraeus, Germany, completed the acquisition of the Fusion UV Systems group headquartered in Gaithersburg, Maryland (USA), on January 31,2013 . With the takeover, Heraeus Noblelight becomes the leading supplier of industrial ultraviolet (UV) process solutions. The enhanced technology portfolio covers all known methods of UV light generation, such as microwave-excited UV lamps and UV light-emitting diode (LED) modules. "The areas of activity and access to markets excellently supplement each other. The acquisition combines development, technology and market competences in an ideal way", explained Rainer Küchler, managing director of the Heraeus Noblelight business group. Heraeus Noblelight now sees itself as being well-placed for the trends in the market of specialty light solutions: the increasing customer demand for integrated process solutions from a single source and the substitution of conventional gas discharge lamps with light-emitting diodes in the medium term.

\section{Strong Exhibitor Interest in PaintExpo 2014}

$M$ ore than 250 exhibitors from 15 countries have already made firm bookings for their booth locations a year in advance of the next PaintExpo. According to the organiser FairFair, the occupied floor space of more than 8,000 square metres already amounts to more than 80 percent of the entire floor space utilised at the event in 2012. This strong early demand by companies from Europe, the USA and Asia confirms the significance attributed to this specialist exhibition as an international presentation and procurement platform. PaintExpo's recipe for success is its exclusive focus on industrial coating technology. In this field, PaintExpo offers a comprehensive range of exhibits, from pretreatment to final inspection. More than 8,000 trade visitors from 63 countries attended the event in 2012. The next PaintExpo will take place from 8 to 11 April 2014 in Karlsruhe, Germany..

\section{Yaskawa Opens New European Headquarters}

$T$ he industrial robot manufacturer Yaskawa opened its new company headquarters in Allershausen near Munich, Germany, on 23 October 2012. The company has invested 12.5 million euros in the new building. The new company headquarters covers an area of around 14,000 square metres and Yaskawa will create around 50 new jobs there. "Allershausen is our centre of expertise for the EMEA countries," said Manfred Stern, President \& COO of Yaskawa Europe, describing the role of the new site at the opening ceremony. From Allershausen, the Ro- botics Division of Yaskawa Europe covers the markets of Europe, Africa and the Middle East and the countries of the former Soviet Union..

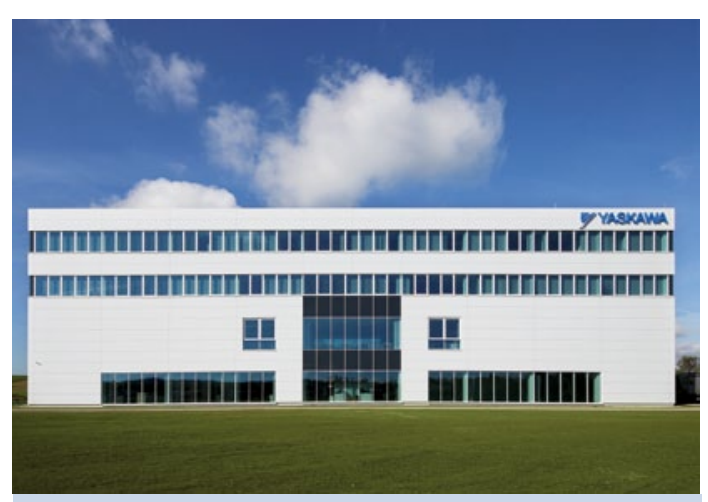

The new European headquarters of Yaskawa in

Allershausen near Munich 